\title{
EFEITO DA FRITURA DE ACARAJÉ NA COMPOSIÇÃO DE CAROTENOIDES E ATIVIDADE ANTIOXIDANTE DE ÓLEO DE PALMA BRUTO
}

\author{
LUCIANA CONCEIÇÃO ARGÔLO CORREIA* \\ SABRINA FEITOSA* \\ DÉBORA BAHIA DE MATOS \\ DEUSDÉLIA TEIXEIRA DE ALMEIDA**** \\ ALESSANDRO DE OLIVEIRA RIOS \\ ITACIARA LARROZA NUNES ${ }^{* *+1+}$
}

\begin{abstract}
O objetivo deste trabalho foi avaliar o efeito da fritura de acarajé nos carotenoides e na atividade antioxidante de óleo de palma bruto (OPB). Foi realizado experimento de fritura de acarajé em OPB, sendo retiradas amostras nos tempos $0,5,10,15,20$ e 25 horas e submetidas à determinação de carotenoides, cor e atividade antioxidante. O óleo inicial apresentou $584,86 \mu \mathrm{g} \mathrm{g}^{-1}$ de carotenoides reduzindo $96,3 \%$ após $25 \mathrm{~h}$ de fritura, com decaimento de $96,3 \%$ e $96,9 \%$ de $\alpha$ - e all-trans- $\beta$-caroteno, respectivamente, formação de produtos de degradação e perda da cor alaranjada. A atividade antioxidante reduziu de 52,78 para $16,63 \%$ e de 28,06 para $2,48 \%$ pelos métodos DPPH e ABTS. Conclui-se que os carotenoides presentes no óleo de palma bruto degradam-se nas primeiras horas de fritura e que a prática de reposição do meio de fritura com óleo usado acentua a inibição da atividade antioxidante.
\end{abstract}

PALAVRAS-CHAVE: ELAEIS GUINEENSIS; ALL-TRANS-B-CAROTENO; COR; CLAE; DPPH; ABTS.

\footnotetext{
* Mestre em Ciência de Alimentos, Faculdade de Farmácia, Universidade Federal da Bahia (UFBA), Salvador, BA (e-mail: luengal@bol.com.br).

** Mestre em Alimentos, Nutrição e Saúde, Escola de Nutrição, UFBA, Salvador, BA (e-mail: sabrinafeitosa0@gmail.com).

*** Graduanda em Nutrição, Escola de Nutrição, UFBA, Salvador, BA (e-mail: debi_bahia@hotmail.com).

**** Doutora em Ciência e Tecnologia de Alimentos, Escola de Nutrição, UFBA, Salvador, BA (e-mail: deliata@uol.com.br).

***** Doutor em Ciência de Alimentos, Universidade Federal do Rio Grande do Sul (UFRGS), Porto Alegre, RS (e-mail: alessandro.rios@ufrgs.br).

****** Doutora em Ciência de Alimentos, Escola de Nutrição, UFBA, Salvador, BA (e-mail: itaciara@yahoo.com).
} 


\section{INTRODUÇÃO}

A fritura por imersão é um método de cocção de alimentos, que utiliza óleos ou gorduras vegetais como meio de transferência de calor, conferindo aos alimentos fritos características sensoriais agradáveis como cor, sabor, textura e aroma. Porém, apesar destas alterações positivas, ocorrem reações que modificam a qualidade funcional e nutricional dos alimentos (CORSINI e JORGE, 2006; SUELIMAN, EL-MAKHZANGY e RAMADAN, 2006).

Durante o processo de fritura, os óleos sofrem alterações provocadas por três agentes: 1) da umidade do alimento - que causa alteração hidrolítica; 2) do oxigênio atmosférico - que provoca oxidação e 3) da elevada temperatura de fritura (aproximadamente $180^{\circ} \mathrm{C}$ ), que provoca a degradação térmica (CORSINI e JORGE, 2006; ANDREU-SEVILLA, 2008).

O óleo de palma bruto (OPB) ou azeite de dendê é empregado na fritura de acarajés, iguaria produzida à base de feijão fradinho descorticado, cebola ralada e sal, sendo este reconhecido como patrimônio imaterial do Brasil desde 2005 e largamente consumido em Salvador-BA (Almeida et al., 2013). A maior diferença entre o óleo de palma e os outros óleos vegetais é sua composição química peculiar: ácidos graxos monoinsaturados $(36,0-45,0 \%)$, polinsaturados $(9,0-12,5 \%)$ e saturados $(43,3-57,4 \%)$, presença de compostos antioxidantes, como tocotrienois e tocoferois (150-1500 mg $\mathrm{Kg}^{-1}$ ) e carotenoides (500-2000 mg Kg-1), o que confere ao mesmo, elevada estabilidade a oxidação (ANDREU-SEVILLA, 2008; CODEX STAN, 2011).

A cor vermelha ou alaranjada do OPB é determinada pela elevada quantidade de carotenoides, sendo o all-trans- $\beta$-caroteno e o all-trans- $\alpha$-caroteno os majoritários (80-90 \%). ${ }^{3}$ Alguns desses compostos possuem atividade de pró-vitamina $A$, além de atuarem como antioxidantes, sequestrando espécies reativas de oxigênio, como o radical peroxil (ROO*) e o oxigênio singlete $\left({ }^{1} \mathrm{O}_{2}\right)$, contribuindo para que resistam mais tempo em temperaturas elevadas (RODRIGUEZAMAYA, 2004; CERQUEIRA, MEDEIROS e AUGUSTO, 2007).

Os carotenoides ocorrem naturalmente na forma trans, sendo que o aquecimento por tempo prolongado e a presença de luz podem conduzir a isomerização para as formas cis, além de propiciar a oxidação que provoca a formação de epóxidos, apocarotenoides e compostos de baixo peso molecular, resultando em perda de cor, de atividade antioxidante e pró-vitamínica $A$ (RODRIGUEZ-AMAYA, 2004; ACHIR et al., 2010; ZEB e MURKOVIC, 2011).

A maior parte dos estudos com óleos vegetais utilizados para a fritura trata da formação de compostos de decomposição lipídica, sem avaliar a degradação dos carotenoides e/ou a redução da atividade antioxidante. Considerando esse fato e a grande utilização do óleo de palma bruto para a fritura de acarajés na Bahia, o objetivo deste estudo foi avaliar a composição dos carotenoides do óleo de palma bruto e o seu comportamento na fritura de acarajés, atividade antioxidante e alteração da cor desse óleo, proporcionando conhecimentos com base na experimentação prática.

\section{MATERIAL E MÉTODOS}

\subsection{AMOSTRA}

Aproximadamente $30 \mathrm{~L}$ de OPB (oleína + estearina), de mesmo lote, foram adquiridos na Feira de São Joaquim, Salvador, Bahia, Brasil. O OPB foi acondicionado em recipiente de aço inoxidável, aquecido e homogeneizado a $45^{\circ} \mathrm{C}$ e retirado alíquota de $250 \mathrm{~mL}$ (tempo 0) e armazenados $\mathrm{a}-20^{\circ} \mathrm{C}$ até análise posterior.

\subsection{EXPERIMENTO DE FRITURA DE ACARAJÉS EM OPB}

A preparação do acarajé foi realizada por uma baiana de acarajé de acordo com a sua prática tradicional, sendo adquiridos diariamente $5 \mathrm{Kg}$ de massa crua, na Feira de São Joaquim. 
A massa de acarajé foi colocada em uma panela de alumínio, batida e utilizada para moldar os bolinhos (60-110 g cada).

Realizou-se o aquecimento de $5 \mathrm{~L}$ de OPB, em um tacho esmaltado, por $12 \mathrm{~min}$. na presença de uma cebola. Em seguida, 5 desses bolinhos de acarajé foram sucessivamente adicionados ao OPB e fritos por imersão, durante aproximadamente $6 \mathrm{~min}$. O tempo total de fritura por dia foi de 5 h, durante cinco dias consecutivos, sempre ao ar livre. Foram fritos aproximadamente 110 acarajés por dia. O óleo ao final da fritura era decantado e armazenado em panela esmaltada (tacho) com tampa à temperatura ambiente.

Ao final de cada dia de fritura foram coletados $250 \mathrm{~mL}$ de OPB, obtendo-se, então, as amostras de 5, 10, 15, 20 e $25 \mathrm{~h}$. A temperatura do OPB foi aferida com termômetro tipo espeto (Incoterm) aos $12 \mathrm{~min}$. iniciais e a cada hora de fritura e a cebola foi reposta sempre que apresentava aspecto de queimada. Todos os dias de fritura começaram com $5 \mathrm{~L}$ de OPB no tacho. No dia 1 , apenas óleo novo foi usado e, nos dias subsequentes, foi utilizado uma mistura do óleo restante do dia anterior (óleo usado) com óleo novo. Quanto à reposição durante a fritura, houve um turnover máximo de $\mathbf{2} L$ por dia, sendo que nos dias 1 e 2 apenas OPB novo foi adicionado, e nos outros dias, a reposição foi realizada com uma mistura aleatória de OPB novo com usado, de acordo com a prática da baiana.

\subsection{CAROTENOIDES TOTAIS}

As amostras foram dissolvidas na proporção de $\cong 0,03-2,0 \mathrm{~g}$ de OPB para $10 \mathrm{~mL}$ de éter de petróleo e os carotenoides totais quantificados em espectrofotômetro UV-Vis (Perkin Elmer, Lambda 25) a $450 \mathrm{~nm}$ e coeficiente de absorção $\left(\mathrm{A}^{1 \%}{ }_{1 \mathrm{~cm}}\right)$ de 2592 (RODRIGUEZ-AMAYA, 2004).

\subsection{EXTRAÇÃO DOS CAROTENOIDES}

A extração dos carotenoides foi realizada segundo Zeb e Murcovic (2011), com acetona gelada $(1: 10, \mathrm{~m}: \mathrm{v})$, agitação em vórtex $(20 \mathrm{~s})$ e armazenamento por $24 \mathrm{~h}$ à $-20^{\circ} \mathrm{C}$, para a remoção da gordura. Os extratos em acetona foram filtrados e os carotenoides transferidos para éter de petróleo:éter etílico (1:1), secos com nitrogênio $\left(\mathrm{N}_{2}\right)$, ressuspendidos em éter tert metil butílico, filtrados em membrana de $0,22 \mu \mathrm{m}$ e analisados por CLAE.

\subsection{ANÁLISE POR CLAE}

As análises por CLAE foram realizadas, segundo Rosso e Mercadante (2007), sendo utilizado cromatógrafo líquido de alta eficiência (Waters, 2695), equipado com detector de arranjo de diodos (DAD) (Waters, modelo 2998), sistema quaternário de bombas (modelo Alliance 2695), degaseificador online e injetor automático.

Os carotenoides foram separados em coluna de fase reversa YMC $C_{30}(250 \times 4,6 \mathrm{~mm}$ d.i, $3 \mu \mathrm{m})$, utilizando como fase móvel gradiente linear de metanol com $0,1 \%$ trietilamina/éter tert metil butílico de 95:5 para 70:30 em 30 min., para 50:50 em 20 min. e mantendo essa proporção até

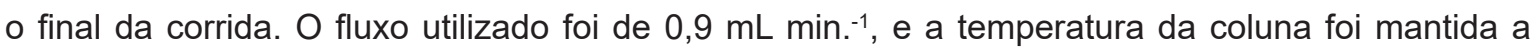
$22^{\circ} \mathrm{C}$, e volume de injeção de $20 \mu \mathrm{L}$. Os espectros UV-Vis foram obtidos entre 250 e $600 \mathrm{~nm}$ e os cromatogramas processados a $450 \mathrm{~nm}$. Os carotenoides foram identificados pela ordem de eluição, co-cromatografia com padrões autênticos de all-trans- $\alpha$ - e all-trans- $\beta$-caroteno (Sigma Aldrich) e características do espectro UV-Vis $\left(\lambda_{\text {máx }}, \%\right.$ III/II e \% $\left.\mathrm{A}_{b} / \mathrm{A}_{\|}\right)$(RODRIGUEZ-AMAYA, 2004; ACHIR et al., 2010; ROSSO e MERCADANTE, 2007).

O método para a quantificação de all-trans- $\alpha$ - e all-trans- $\beta$-caroteno foi validado quanto à linearidade, Limite de Detecção (LD), Limite de Quantificação (LQ) e precisão (intra e interdias) segundo Silva et al., (2011). As curvas de calibração foram construídas com seis níveis de concentrações crescentes de solução padrão de $\alpha$-caroteno $\left(0,05-10 \mu \mathrm{g} \mathrm{mL}^{-1}\right)$ e de $\beta$-caroteno $(0,04$ 
a $8,00 \mu \mathrm{g} \mathrm{mL}^{-1}$ ). Foi feita três repetições em dias diferentes, sendo a equação da reta e o coeficiente de linearidade determinados através de regressão linear (SILVA et al., 2011).

A precisão inter e intra-dias, foram determinadas no mesmo dia e em dias diferentes, respectivamente, em triplicata, a partir da injeção das soluções de menor, intermediária e de maior concentração - $\alpha$-caroteno $\left(0,04,2,00\right.$ e $\left.8,00 \mu \mathrm{g} \mathrm{mL}^{-1}\right)$ e $\beta$-caroteno $\left(0,05,2,50\right.$ e $\left.10,00 \mu \mathrm{g} \mathrm{mL}^{-1}\right)$-. A precisão do método analítico foi expressa em função do desvio padrão relativo $(\% \mathrm{CV})$.

\subsection{ENSAIOS DA ATIVIDADE ANTIOXIDANTE PELOS MÉTODOS DPPH E ABTS}

A atividade antioxidante das amostras de óleo de palma bruto submetido à fritura (nos tempos $0,5,10,15,20,25 \mathrm{~h}$ ) foi determinada pelos métodos DPPH e ABTS. O ensaio antioxidante pelo método DPPH foi realizada de acordo com a metodologia proposta por Kalantzakis et al., (2006). Para a realização das medições da atividade antioxidante $\cong 100 \mathrm{mg}$ de óleo de palma foi adicionado a $4000 \mu \mathrm{L}$ de solução de $\mathrm{DPPH}^{\cdot}(100 \mu \mathrm{M})$ em acetato de etila, homogeneizada vigorosamente e deixada no escuro. A leitura da absorbância da mistura foi mensurada em espectrofotômetro UV-Vis após 30 min. de reação a $515 \mathrm{~nm}$.

A atividade antioxidante determinada pelo método ABTS foi realizada segundo Karaosmanoglu et al., (2010). Para isso, $5 \mathrm{~mL}$ de $n$-hexano foram adicionados a $\cong 25 \mathrm{mg}$ de óleo de palma, depois uma alíquota de $10 \mu \mathrm{L}$ desta solução foi adicionada a $1 \mathrm{~mL}$ da solução do radical ABTS $^{+*}$. A leitura foi feita após 6 min. de reação em espectrofotômetro UV-Vis, a $734 \mathrm{~nm}$.

Os resultados de ambos os experimentos foram expressos como porcentagem de inibição, de acordo com a equação 1:

$$
\text { \%_inibição= }(\text { Ac-As }) / A c \times 100 \%
$$

\section{Equação 1}

Onde: As= absorbância da amostra e Ac= absorbância do radical $\mathrm{DPPH}^{*}$ ou $\mathrm{ABTS}^{+*}$

\subsection{COR}

As análises foram realizadas em colorímetro Minolta (CR 400), com iluminante $D_{65}$, ângulo de observação de $2^{\circ}$, diâmetro da fenda de $11 \mathrm{~mm}$ e escala CIELab: $L^{*}$ (luminosidade) que varia entre o branco e o preto, sendo que 0 (zero) corresponde ao preto/escuro e o 100 (cem) ao branco, $a^{*}$ (intensidade de vermelho): variando de verde (-a) a vermelho (+a), b* (intensidade de amarelo): variando de azul (-b) a amarelo (+b)], $C^{*}$ (Chroma): $\left(a^{* 2}+b^{* 2}\right)^{1 / 2}$, ângulo hab (arco tangente): $b^{*} / a^{*}$ (ANDREU-SEVILLA et al., 2008).

\subsection{ANÁLISE ESTATÍSTICA}

Todas as análises foram realizadas em triplicata e os resultados avaliados pelo teste de comparação de médias de STEEL (1960), $(p<0,05)$ usando o programa "R" 2.15.3. O teste de Spearman avaliou a correlação entre os carotenoides totais com a cor e a atividade antioxidante.

\section{RESULTADOS E DISCUSSÃO}

O teor médio de carotenoides totais encontrado no OPB $\left(0 \mathrm{~h}\right.$ ) foi de $584,86 \mu \mathrm{g} \mathrm{g}^{-1}$ (Tabela 1), este valor foi similar aos observados por Almeida et al., (2013), que encontraram valores de $526,77 \mu \mathrm{g} \mathrm{g}^{-1}$. Já em um experimento de fritura de batatas, utilizando óleo de palma Sioma ${ }^{\circledR}$, Andreu-Sevilla et al., (2008), verificaram um menor teor inicial de carotenoides totais $\left(481 \mu \mathrm{g} \mathrm{g}^{-1}\right)$. Cabe ressaltar que as diferenças nos teores de carotenoides em OPB podem ser influenciadas por diferentes variedades e grau de amadurecimento dos frutos, processo de extração, armazenamento e proporção de estearina e oleína (CURVELO et al., 2011). 


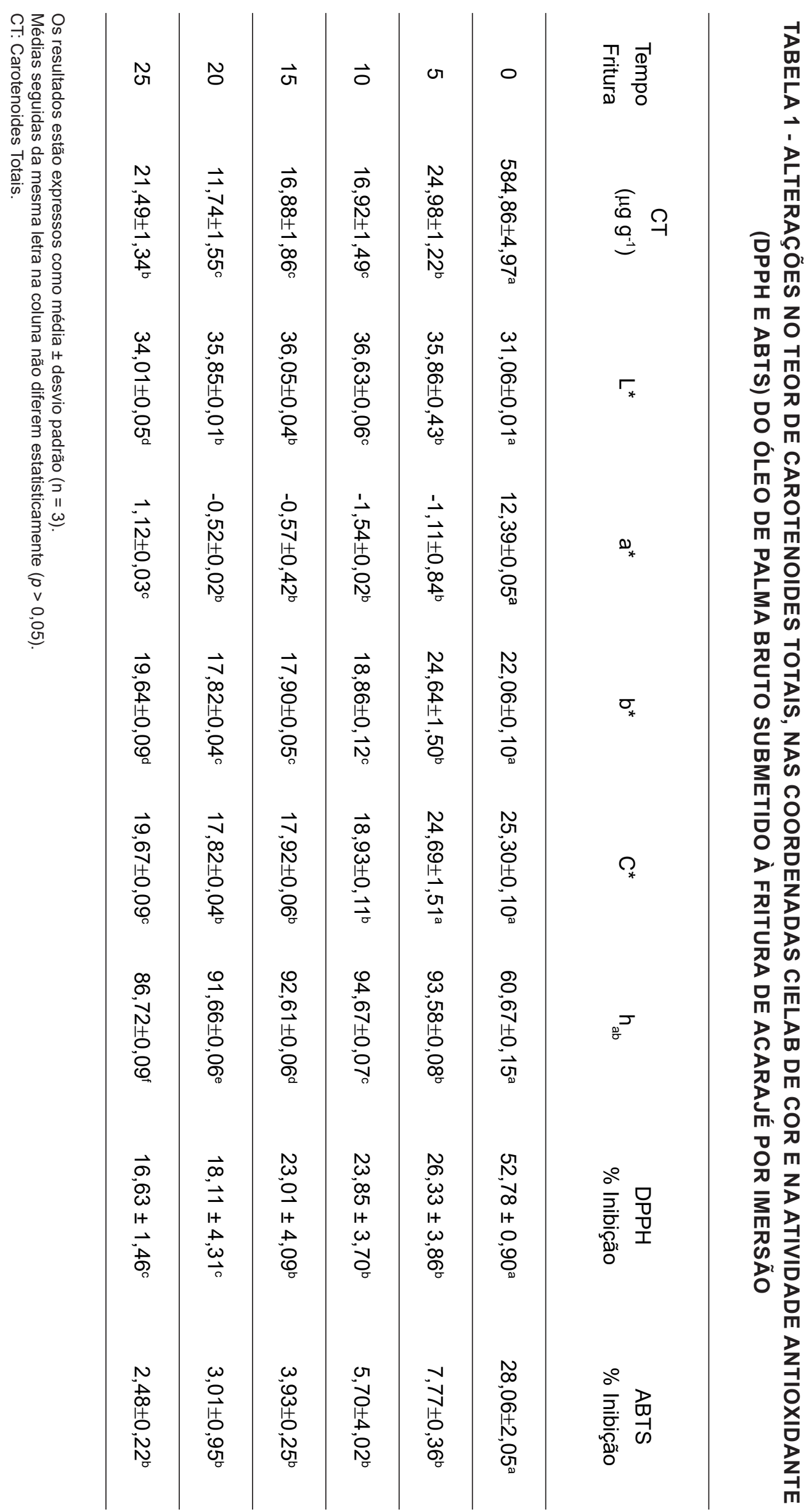


No decorrer da fritura, utilizando-se uma temperatura média de $161^{\circ} \mathrm{C}$ foi observada uma redução significativa no teor de carotenoides totais em relação ao conteúdo inicial $(p<$ 0,05 ), sendo perdidos $95,73 \%$ dos mesmos em $5 \mathrm{~h}$ de fritura. No estudo de Andreu-Sevilla et al., (2008), estes verificaram reduções de $52,2 \%$ e de $97,2 \%$ do conteúdo de carotenoides totais do óleo de palma Sioma ${ }^{\circledR}$ submetido à fritura a $180^{\circ} \mathrm{C}$ e $240^{\circ} \mathrm{C}$, respectivamente durante 90 min.

Tais alterações podem ser atribuídas à degradação dos carotenoides durante o processo de fritura, como também ao resultado da exposição do óleo a fatores como o oxigênio, luz, elevadas temperaturas, moléculas pró-oxidantes, que podem ocasionar a isomerização e oxidação destes pigmentos (ANDREU-SEVILLA et al., 2008). Outro fator que pode estar contribuinado para a redução destes pigmentos, durante a fritura, pode ser devido à incorporação dos mesmos pelos alimentos fritos (MANORAMA e RUKMINI, 1991).

Para todos os tempos estudados, o teor de carotenoides sempre se manteve baixo $11,74-24,98 \mu \mathrm{g} \mathrm{g}^{-1}$, comparando-se ao valor inicial (Tabela 1). Cabe destacar, que o tempo de $5 \mathrm{~h}$ apresentou a maior concentração destas moléculas, em relação aos tempos 10, 15, 20 e $25 \mathrm{~h}$, provavelmente pelo emprego apenas de óleo novo no processo de fritura no primeiro dia.

As coordenadas $L^{*}, a^{*}, b^{*}$ e $h_{a b}$ do óleo inicial, apresentaram diferença estatística significativa em relação aos demais tempos de fritura (Tabela 1). De modo geral o óleo foi se tornando mais luminoso (aumento de $L^{*}$ ), menos vermelho (redução de $a^{*}$ ) e menos amarelo (redução de $b^{*}$ ). Os valores de $C^{*}$ não diferiram estatisticamente nos tempos 0 e $5 \mathrm{~h}$, mas apresentaram diferença em relação aos demais tempos. Somente as coordenadas $b^{*}(r=$ $0,94)$ e $C^{*}(r=0,99)$ apresentaram correlação significativa positiva com os carotenoides totais, indicando que a perda da cor laranja é decorrente das alterações como também das perdas dos carotenoides.

Curvelo et al., (2011) demostraram valores superiores para todos os parâmetros de cor de OPB empregados em frituras de acarajés, quando comparados aos valores obtidos nas $5 \mathrm{~h}$ de fritura deste estudo, tais diferenças podem ser relacionadas aos diferentes tempos e técnicas de fritura empregada no referido estudo.

As porcentagens de inibição para os radicais $\mathrm{DPPH}^{\bullet}$ e $\mathrm{ABTS}^{+\bullet}$ do óleo foram reduzidas significativamente com o tempo de fritura $(p<0,05)$ (Tabela 1), não ocorrendo correlação significativa entre percentual de inibição (DPPH: $r=0,65 ; p>0,05$; ABTS: $r=0,60 ; p>0,05$ ) e carotenoides totais. Kalantzakis et al., (2006) ao aquecerem blend (de óleo de palma refinado, óleo de algodão e óleo de canola) por $10 \mathrm{~h}$ a $180^{\circ} \mathrm{C}$, encontraram resultado superior $(77,3 \%)$ para o percentual de inibição do radical DPPH', provavelmente devido as diferentes condições experimentais (solvente, tempo de reação, quantidade de amostra e concentração da solução de DPPH) e/ou conteúdo de antioxidantes (KARAOSMANOGLU et al., 2010).

Também foi observado menor inibição do radical ABTS ${ }^{+\bullet}$ nos tempos 5 e $10 \mathrm{~h}$, quando comparado aos tempos 15, 20 e $25 \mathrm{~h}$ de fritura (Tabela 1). Isso pode ter ocorrido provavelmente pela utilização de óleo novo no início da fritura (Tempo $5 \mathrm{~h}$ ) e na reposição (Tempos 5 e $10 \mathrm{~h}$ ). Porcentagens de inibição do radical $\mathrm{ABTS}^{+\bullet}$ inferiores ao verificado neste estudo para o OPB (0 h) $(28,06 \%)$, foram encontradas por Karaosmanoglu et al., (2010) para óleos de canola $(22,52 \%)$, avelã $(21,19 \%)$ e azeite de oliva refinado $(23,50 \%)$, provavelmente pelo maior teor de carotenoides (500-2000 mg Kg-1), vitamina E no OPB (150-1500 mg Kg-1) e adição de cebola ao meio de fritura (CODEX STAN, 2011; FELZENSZWALB et al., 2014). Sendo assim, a atividade antioxidante do OPB pode estar relacionada principalmente ao elevado conteúdo de vitamina $E$ presente no mesmo, como também devido a sua composição de ácidos graxos (monoinsaturados, polinsaturados e saturados) e a prática da baiana de acarajé de adicionar cebola ao OPB (CODEX STAN, 2011; FELZENSZWALB et al., 2014).

O método utilizado para a quantificação de all-trans- $\alpha$-caroteno e all-trans- $\beta$-caroteno 
apresentou boa linearidade $(R=0,9931$ e 0,9986$)$, respectivamente. O LD e o LQ para o all-trans$\alpha$-caroteno foram de $22,8 \mathrm{ng} \mathrm{mL}^{-1}$ e $69,0 \mathrm{ng} \mathrm{mL}^{-1}$ e para o all-trans- $\beta$-caroteno foram de $38,5 \mathrm{ng} \mathrm{mL}^{-1}$ e $111,5 \mathrm{ng} \mathrm{mL}^{-1}$. Os valores médios de Coeficiente de Variação $(\% \mathrm{CV})$ para a precisão intra e interdias foram de 4,3\% e 3,0\% para all-trans- $\alpha$-caroteno e de 5,7\% e 4,0\% para all-trans- $\beta$-caroteno, de acordo com limite permitido (até 20\%) (SILVA et al., 2011).

Silva et al., (2011) obtiveram correlação $(0,9942)$, LD (50 $\left.\mathrm{ng} \mathrm{mL}^{-1}\right), \mathrm{LQ}\left(100 \mathrm{ng} \mathrm{mL}^{-1}\right)$, precisão intra-dia $(\% C V=5,15)$ e precisão inter-dias $(\% C V=9,26)$ superiores, ao validarem método para determinação de all-trans- $\beta$-caroteno em óleos de buriti, tucumã e patuá, devido a diferenças entre equipamentos, analistas e condições de validação.

No OPB (0 h) (Tabela 2, Figura 1), foram identificados 11 carotenoides. Andreu-Sevilla et al., (2008) identificaram 9 carotenoides em óleo de palma Sioma ${ }^{\circledR}$, sendo quatro destes similares aos do OPB (0 h) (luteína, all-trans- $\alpha$-caroteno, all-trans- $\beta$-caroteno e 9-cis- $\beta$-caroteno). A diferença na composição de carotenoides se deve a variedade da palma, grau de amadurecimento dos frutos e extração e armazenamento (ANDREU-SEVILLA et al., 2008).

\section{TABELA 2 - CARACTERÍSTICAS DOS PRINCIPAIS CAROTENOIDES DE ÓLEO DE PALMA BRUTO}

\begin{tabular}{|c|c|c|c|c|c|}
\hline Pico & Carotenoide $^{a}$ & $\operatorname{Tr}$ (minutos) $^{\mathrm{b}}$ & $\lambda_{\text {máx }}(\mathrm{nm})$ & $\%$ III/II & $\% A_{B} / A_{\|}$ \\
\hline 1 & 13 -cis- $\alpha$-caroteno ${ }^{d}$ & $24,5-25,3$ & $332,418,440,466$ & 28 & 46 \\
\hline 2 & cis-luteína & $25,8-26,0$ & $330,412,435,460$ & 23 & 20 \\
\hline 3 & di-cis- $\alpha$-caroteno ${ }^{d}$ & $26,3-26,7$ & $330,415,436,462$ & 23 & 33 \\
\hline 4 & Não identificado & $27,3-28,4$ & $333,410,436,456$ & 0 & 41 \\
\hline 5 & 13 -cis- $\beta$-caroteno ${ }^{d}$ & $28,7-29,1$ & $338,422,443,466$ & 9 & 43 \\
\hline 6 & di-cis- $\beta$-caroteno ${ }^{d}$ & $29,4-30,1$ & $337,420,443,470$ & 9 & 33 \\
\hline 7 & all-trans- $\alpha$-caroteno ${ }^{\mathrm{e}}$ & $31,4-31,8$ & $422,445,473$ & 55 & 0 \\
\hline 8 & 9 -cis- $\alpha$-caroteno ${ }^{d}$ & $32,7-33,0$ & $330,418,440,469$ & 62 & 9 \\
\hline 9 & Não identificado & $35,0-35,4$ & $422,440,467$ & 33 & 0 \\
\hline 10 & all-trans- $\beta$-caroteno ${ }^{\mathrm{e}}$ & $35,9-36,3$ & $425,451,478$ & 25 & 0 \\
\hline 11 & 9-cis- $\beta$-caroteno ${ }^{d}$ & $37,8-38,1$ & $341,420,446,473$ & 27 & 11 \\
\hline
\end{tabular}

aNumerados de acordo com a Figura 1 (T Oh).

${ }^{\mathrm{b} T}$ Tempo de retenção. 


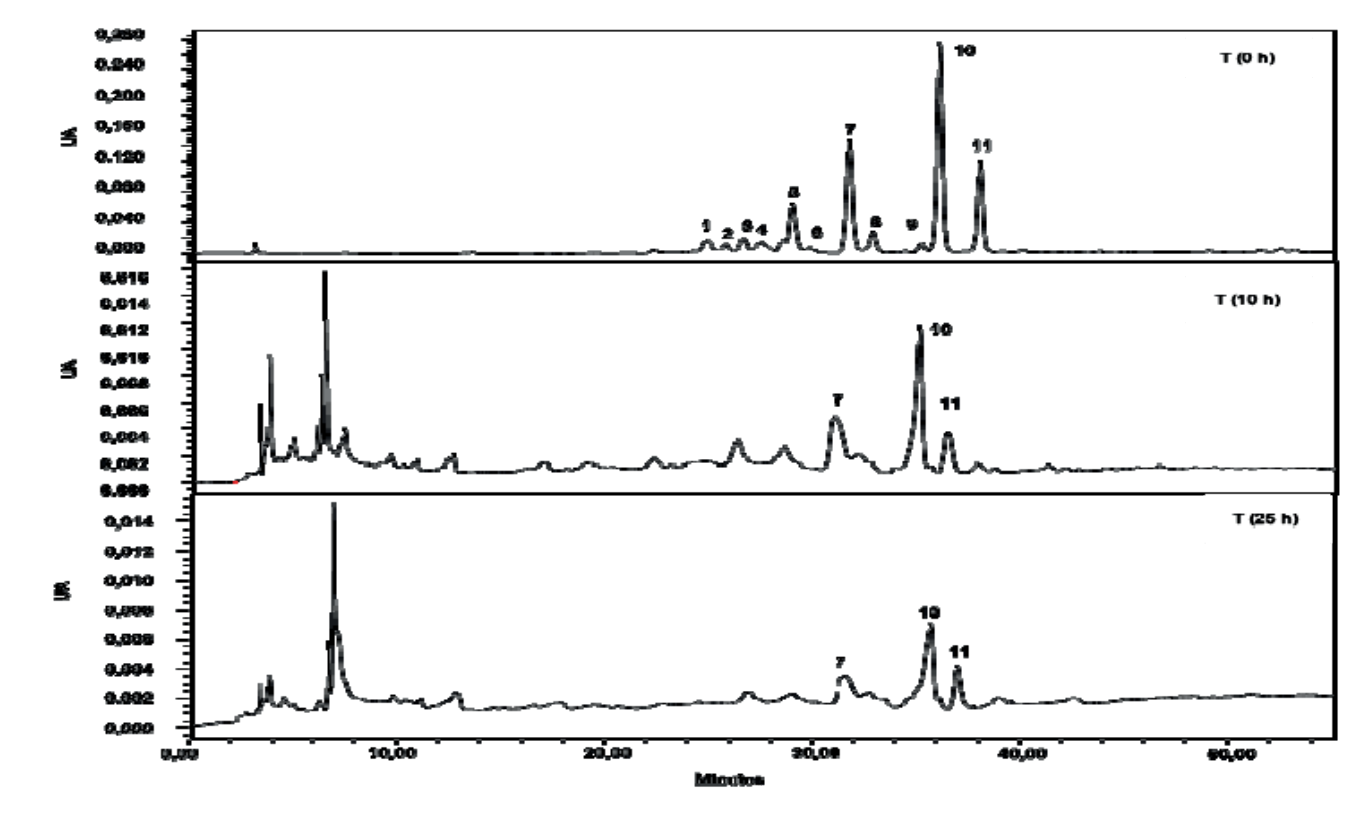

\section{FIGURA 1 - CROMATOGRAMAS, OBTIDOS POR CLAE A 450NM, DE EXTRATOS DE CAROTENOIDES DE ÓLEO DE PALMA BRUTO NOS TEMPOS 0, 10 E 25 H}

Os carotenoides majoritários no OPB inicial foram o all-trans- $\beta$-caroteno e o all-trans$\alpha$-caroteno (Tabela 2, Figura 1), representando 61,3\% (358,5 $\left.\mu \mathrm{g} \mathrm{g}^{-1}\right)$ e $36,0 \%\left(210,6 \mu \mathrm{g} \mathrm{g}^{-1}\right)$ dos carotenoides totais, sendo identificados ainda isômeros cis destes dois carotenos (Tabela 2). Valor similar $\left(367 \mu \mathrm{g} \mathrm{g}^{-1}\right)$ para o all-trans- $\beta$-caroteno, foi observado por Manorama e Rukmini, (1991) em óleo de palma bruto.

Óleo de palma refinado analisado por Rosso e Mercadante (2007), também apresentou esses carotenoides como principais, representando $65,77 \%$ e $22,34 \%$, respectivamente, sendo observada também a presença de epóxidos, que não foram verificados nesse estudo, provavelmente porque a há maior formação desses compostos ocorre durante o processo de refino (LODY, 2009).

No óleo de palma Sioma ${ }^{\circledR}$ avaliado por Andreu-Sevilla et al., (2008), o all-trans- $\alpha$-caroteno foi responsável por $47 \%$ e o all-trans- $\beta$-caroteno por apenas $17 \%$, além disso, estes identificaram $\gamma$-caroteno, $\varepsilon$-caroteno e $\delta$-caroteno, não encontrados no OPB avaliado nesse estudo, indicando uma composição diferente de carotenoides na variedade de palma Sioma, em relação à palma cultivada no Brasil (ANDREU-SEVILLA et al., 2008; LODY, 2009).

All-trans- $\beta$-caroteno e all-trans- $\alpha$-caroteno sofreram redução com o tempo de fritura (Figuras 1 e 2), atingindo 96,3\% e 96,9\% após 25 h, respectivamente (Figura 2). Considerando que somados os mesmos representam $97,3 \%$ dos carotenoides no OPB e são os principais carotenoides com atividade de pró-vitamina $A$, é importante destacar a elevada perda vitamínica em $5 \mathrm{~h}$ de fritura (RODRIGUEZ-AMAYA e KIMURA, 2004). Ao utilizarem a temperatura de $180^{\circ} \mathrm{C}$ (superior a desse trabalho), Achir et al., (2010) observaram perda de $90 \%$ de all-trans- $\beta$-caroteno de oleína de palma em apenas $30 \mathrm{~min}$. de aquecimento.

Somente os picos correspondentes ao all-trans- $\alpha$-caroteno, all-trans- $\beta$-caroteno 9 -cis- $\beta$ caroteno (Figura 1) mantiveram os espectros similares em todos os tempos avaliados, os demais foram sofrendo modificações. Na presença de temperatura e de oxigênio, os carotenoides são primeiramente isomerizados, depois oxidados (epóxidos) e por último, clivados (apocarotenoides), sendo possível que nos estudos de Andreu-Sevilla et al., (2008) e Achir et al., (2010), um maior número de carotenoides tenha sido identificado pelo menor tempo de aquecimento empregado e/ou menor reação dos carotenoides com os peróxidos formados com a degradação dos lipídeos. 


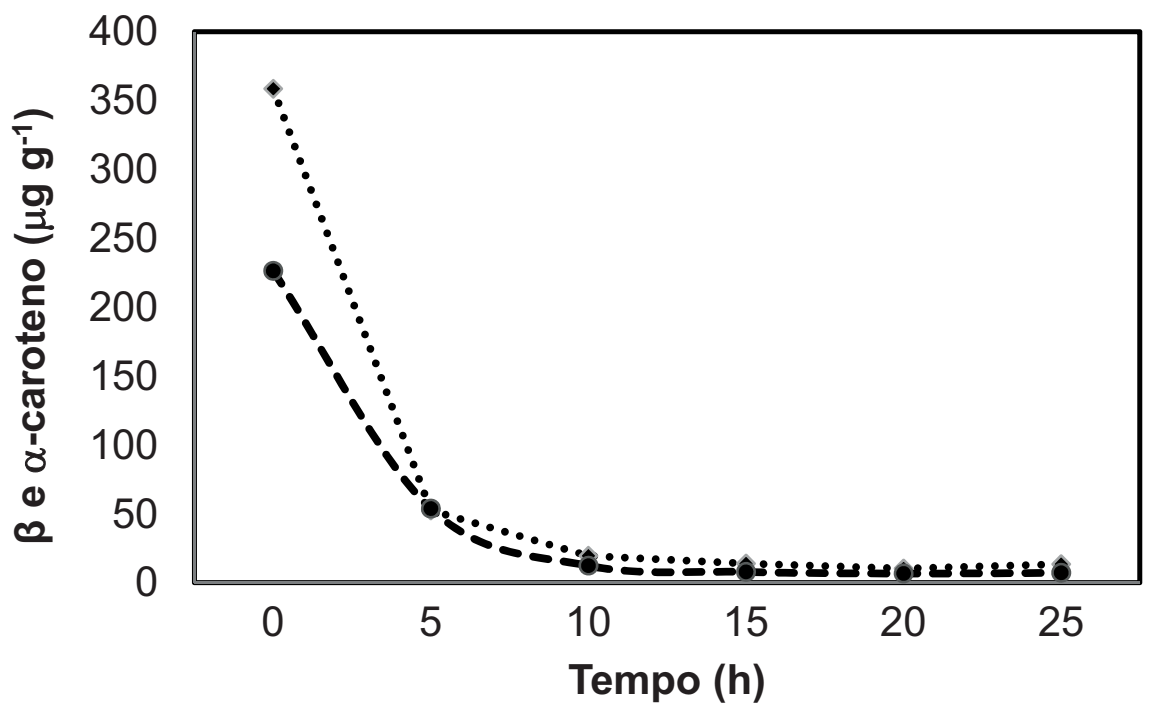

\section{FIGURA 2 - ALTERAÇÕES NAS CONCENTRAÇÕES DE ALL-TRANS-A-CAROTENO (•) E DE ALL-TRANS-B-CAROTENO ( ) NO ÓLEO DE PALMA NO DECORRER DAS 25 H DE FRITURA}

\section{CONCLUSÃO}

Conclui-se que os carotenoides presentes no óleo de palma bruto degradam-se nas primeiras horas de fritura e que a prática de reposição do meio de fritura com óleo usado acentua a inibição da atividade antioxidante. Como continuidade desse trabalho sugere-se um estudo para quantificação dos carotenoides absorvidos pelo acarajé durante a fritura e estudo sobre a ação da vitamina E no óleo de palma bruto.

\section{ABSTRACT
THE EFFECT OF DEEP-FRIED AKARA ON THE COMPOSITION OF CAROTENOIDS AND THE ANTIOXIDANT ACTIVITY OF CRUDE PALM OIL

The objective of this work was to evaluate the effect of frying akara on the carotenoids and on the antioxidant activity of the crude palm oil (CPO). An experiment was performed, frying the akara in CPO and removing samples at $0,5,10,15,20$ and $25 \mathrm{~h}$. They were then submitted to determination of carotenoids, color and antioxidant activity. The initial oil presented 584.86 $\mu \mathrm{g} \mathrm{g}^{-1}$ carotenoids, reducing $96.3 \%$ after $25 \mathrm{~h}$ of frying, with a decrease of $96.3 \%$ and $96.9 \%$ of $\alpha$ - and all-trans- $\beta$-carotene, respectively, as well as formation of degrading products and loss of the orange color. The antioxidant activity reduced from 52.78 to $16.63 \%$ and from 28.06 to $2.48 \%$ by the DPPH and ABTS methods. It was concluded that the carotenoids present in the crude palm oil degrade in the first hours of frying and the practice of replacement the frying medium with a mixture of new and used oil used accentuates the inhibition of antioxidant activity.

KEY-WORDS: ELAEIS GUINEENSIS; ALL-TRANS-B-CAROTENE; COLOR; HPLC; DPPH; ABTS. 


\section{REFERÊNCIAS}

ACHIR, N.; RANDRIANATOANDRO, V. A.; BOHUON, P.; LAFFARGUE, A.; AVALLONE, S. Kinetic study of $\beta$-carotene and lutein degradation in oils during heat treatment. European Journal of Lipid Science and Technology, v. 112, p. 349$361,2010$.

ALMEIDA, D. T. de; NUNES, I. L.; CONDE, P. L.; ROSA, R. P. S.; ROGÉRIO, W. F.; MACHADO, E. R. A quality assessment of crude palm oil marketed in Bahia, Brazil. Grasas y Aceites, v. 64, p. 387-394, 2013.

ANDREU-SEVILLA, A.; HARTMANN, A.; SAYAS, E.; BURLÓ-CARBONELL, F.; DELGADO-ESTRELLA, P.; VALVERDE, J. M.; CARBONELL-BARRACHINA, Á. A. Mathematical quantification of total carotenoids in Sioma oil using color coordinates and multiple linear regression during deep-frying simulations. European Food Research and Technology, v. 226, p. 1283-1291, 2008.

CERQUeIRA, F. M.; MEDEIROS, M. H. G.; AUGUSTO, O. Antioxidantes dietéticos: controvérsias e perspectivas. Química Nova, v. 30, n. 2, p. 441-449, 2007.

CODEX ALIMENTARIUS (FAO/WHO). Codex Standard for named vegetable oils. CODEX STAN 210, 2011. Roma, 2011.

CORSINI, M. S.; JORGE, N.. Estabilidade oxidativa de óleos vegetais utilizados em frituras de mandioca palito congelada. Ciência e Tecnologia de Alimentos, vol. 26, p. 27-32, 2006.

CURVELO, F. M.; ALMEIDA, D. T. DE; NUNES, I. L.; FEITOSA, S. Qualidade do óleo de palma bruto (Elaeis guineensis): matéria-prima para fritura de acarajés. Revista Instituto Adolfo Lutz, v. 70, p. 641-646, 2011.

FELZENSZWALB, I.; COSTA MAZZEI, J. L. DA; FEITOSA, S.; FORTES AIUB, C. A.; ALMEIDA, D. T. de. Toxicological assessment of crude palm oil (Elaeis guineensis) used in deep frying of akara (cowpea paste finger food). Grasas $y$ Aceites, v. 65, p. 1-8,2014.

KALANTZAKIS, G.; BLEKAS, G.; PEGKLIDOU, K.; BOSKOU, D. Stability and radical-scavenging activity of heated olive oil and other vegetable oils. European Journal of Lipid Science and Technology, v.108, p. 329-335, 2006.

KARAOSMANOGLU, H.; SOYER, F.; OZEN, B.; TOKATLI, F. Antimicrobial and Antioxidant Activities of Turkish Extra Virgin Olive Oils. Journal of Agricultural and Food Chemistry, v. 58, p. 8238-8245, 2010.

Lody, R. Dendê símbolo e sabor. Editora SENAC: São Paulo, 2009.

MANORAMA, R.; RUKMINI, C. Effect of processing on $\beta$-carotene retention in crude palm oil and its products. Food Chemistry, v. 42, p. 253-264, 1991.

RODRIGUEZ-AMAYA, D. B.; KIMURA, M. Harvest plus handbook for carotenoid analysis. Washington, DC e Cali: International Food Policy Research Institute e International Center for Tropical Agriculture, 2004, p. 58.

ROSSO, V. V.; MERCADANTE, A. Z. Identification and quantification of carotenoids, by HPLC-PDA-MS/MS, from Amazonian fruits. Journal of Agricultural and Food Chemistry, v. 55, p. 5062-5072, 2007.

SILVA, S. M.; ROCCO, S. A.; SAMPAIO, K. A.; TAHAM, T.; SILVA, L. H. M. DA; CERIAN, R.; MEIRELLES, A. J. A. Validation of a method for simultaneous quantification of total carotenes and tocols in vegetable oils by HPLC. Food Chemistry, $v$. 129, p. 1874-1881, 2011.

STEEL, R. G. D. A rank sum test for comparing all pairs of treatments. Technometrics, v.3, p.197-207, 1960.

SULIEMAN, A. E. R. M.; EL-MAKHZANGY, A.; RAMADAN, M. F.. Antiradical performance and physicochemical characteristics of vegetable oils upon frying of french fries: a preliminary comparative. Electronic Journal of Environmental Agricultural and Food Chemistry, vol. 5, p. 1429-1441, 2006.

ZEB, A.; MURKOVIC, M. Determination of thermal oxidation and oxidation products of $\beta$-carotene in corn oil triacylglycerols. Food Research International, v. 50, p. 534-544, 2011.

\section{AGRADECIMENTOS}

Os autores agradecem ao CNPq pelo apoio financeiro (Processo $n^{\circ} 482790 / 2010-5$ ) e a CAPES pelas bolsas de mestrado concedidas a primeira e segunda autora. 